\title{
In-situ Transmission Electron Microscopy Study of Oxygen Vacancy Ordering and Dislocation Annihilation in Undoped and Sm-doped CeO2 Ceramics During Redox Processes
}

\author{
Yong Ding ${ }^{1}$, Yu Chen ${ }^{1}$, Ken C. Pradel ${ }^{1}$, Meilin Liu ${ }^{1}$ and Zhong Lin Wang ${ }^{1}$
}

1. School of Materials Science and Engineering, Georgia Institute of Technology, Atlanta, GA, United States

Ceria $\left(\mathrm{CeO}_{2}\right)$ based ceramics have been widely used for many applications due to their unique ionic, electronic, and catalytic properties. Here we report our findings in investigating into the redox processes of undoped and $\mathrm{Sm}$-doped $\mathrm{CeO}_{2}\left(\mathrm{Sm}_{0.2} \mathrm{Ce}_{0.8} \mathrm{O}_{1.9}\right.$, SDC) ceramics stimulated by high-energy electron beam irradiation within a transmission electron microscope (TEM) [1].

The reduced structure with oxygen vacancy ordering has been identified as the $\mathrm{CeO}_{1.68}\left(\mathrm{C}-\mathrm{Ce}_{2} \mathrm{O}_{3+\delta}\right)$ phase via high-resolution TEM (HRTEM). Figure 1 gives the HRTEM images from the same SDC grain projected along different orientations. While the insets are simulated images using standard $\mathrm{CeO}_{1.68}$ structure, which match the experimental ones pretty well. The reduction of $\mathrm{Ce}^{4+}$ to $\mathrm{Ce}^{3+}$ has been monitored by electron energy-loss spectroscopy. The decreased electronic conductivity of the SDC is revealed by electron holography, as positive electrostatic charges accumulated at the surfaces of SDC grains under electron beam irradiation, but not at $\mathrm{CeO}_{2}$ grains.

The formation of the reduced $\mathrm{CeO}_{1.68}$ domains corresponds to lattice expansion compared to the $\mathrm{CeO}_{2}$ matrix [2]. Therefore the growth of $\mathrm{CeO}_{1.68}$ nuclei builds up strain inside the matrix, causing annihilation of dislocations inside the grains. By using in-situ high-resolution TEM and a fast OneView camera recording system, we investigated dislocation motion inside both $\mathrm{CeO}_{2}$ and SDC grains under electron beam irradiation. As displayed in Figure 2, the dislocation prefers to dissociate into Shockley partials bounded by stacking faults. Then the partials can easily glide in one of the $\{111\}$ planes to reach the grain surfaces. Even the Lomer-Cottrell lock can be swept away by the phase change induced strain field. Our results revealed the high mobility of dislocations inside $\mathrm{CeO}_{2}$ and SDC grains during their respective redox processes [3].

\section{References:}

[1] Y Ding et al, Journal of Applied Physics 120 (2016), p. 214302.

[2] E A Kummerle and G Heger, Journal of Solid State Chemistry 147 (1999), p. 485.

[3] The authors acknowledge the support from the Hightower chair foundation, the National Science Foundation (DMR-1505319), and the US Department of Energy ARPA-E REBELS Program (DEAR0000502). 

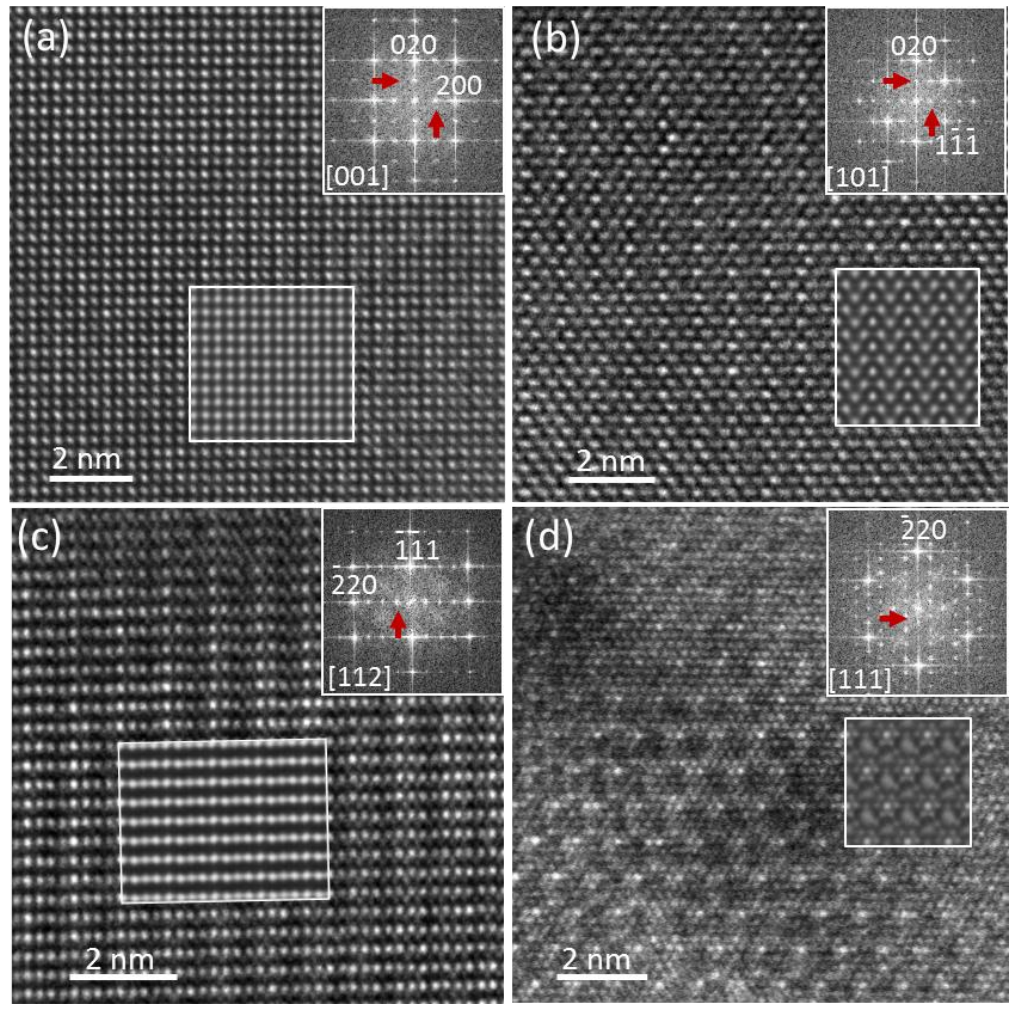

Figure 1. HRTEM images of SDC along different incident electron beam directions: (a) [001]; (b) [101]; (c) [112]; (d) [111]. The FFT patterns and simulated HRTEM images are inserted.

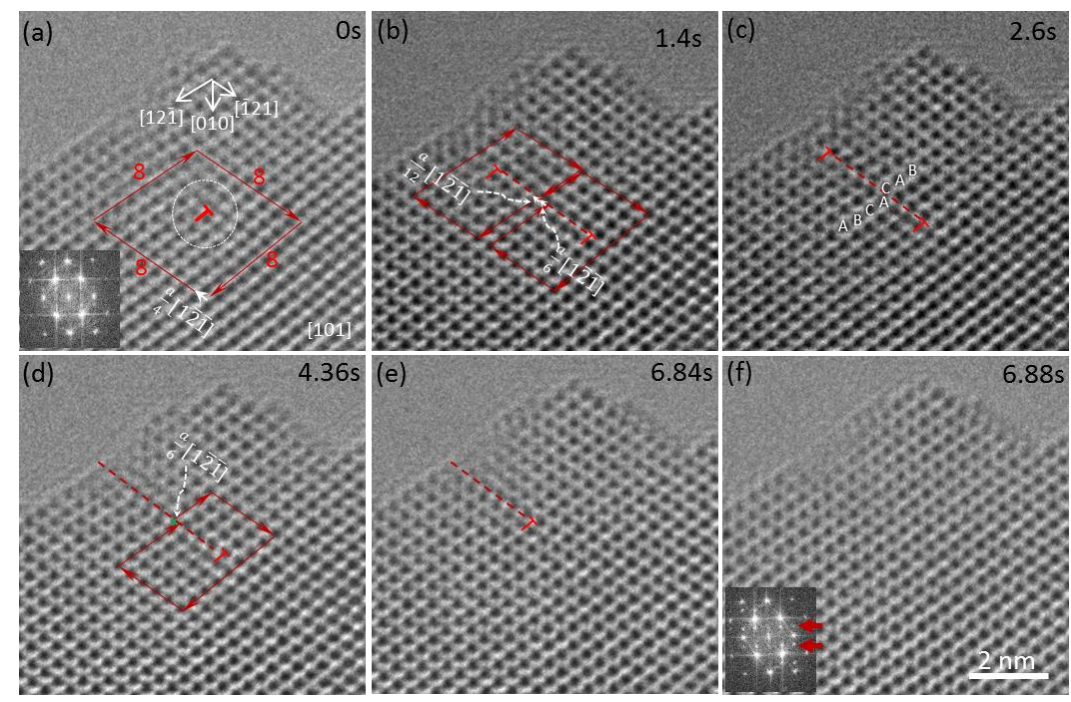

Figure 2. A series of HRTEM images recorded in time sequence from the same area of a $\mathrm{CeO} 2$ grain. 